\title{
Maldición de la abundancia, pueblos aislados y economía extractiva en Perú y Ecuador
}

Robert Wasserstrom*

\begin{abstract}
RESUMEN
En Ecuador y Perú, Se incrementan los contactos entre los grupos indígenas aislados y La gente de

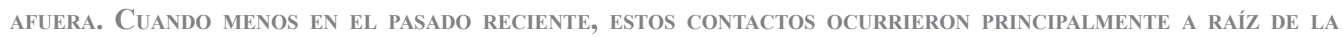
EXPloración Petrolera Que Se llevaba a Cabo en Varias zonas de la Región amazónica. Sin embargo, es un ERROR CONSIDERAR A LAS EMPRESAS COMO PRINCIPALES PROTAGONISTAS DE LOS PROBLEMAS SURGIDOS EN MUCHAS zoNAS de la CUENCa AMAZÓNICA. Al CONTRARIO, AQUí SE EXAMINA EL TEMA DEL CONTACTO DESDE LA PERSPECTIVA DE LA "MALDICIÓN DE LOS RECURSOS," ES DECIR, COMO CONSECUENCIA DE UN SISTEMA ELABORADO POR LAS ELITES NACIONALES PARA APROPIARSE DE LA RIQUEZA PETROLERA "SIN EL CONSENTIMIENTO DE LOS CIUDADANOS". EN ESTE SISTEMA, LAS EMPRESAS PETROLERAS - SEAN PRIVADAS O PÚBLICAS - FUNCIONAN PRINCIPALMENTE COMO AGENTES O COMO CÓMPLICES, PERO RARAMENTE COMO PROTAGONISTAS DE LAS POLÍTICAS EXTRACTIVAS. TAMBIÉN SE DOCUMENTA EL REDUCIDO PAPEL ACTUAL DE LA INDUSTRIA PETROLERA EN LOS TERRITORIOS DE PUEBLOS AISLADOS, SOBRE TODO EN Perú, donde estos pueblos ahora enfrentan algunos Peligros más graves Como el negocio CRIMinal de La MADERA, LA COCA Y EL ORO.
\end{abstract}

Palabras claves: Pueblos indígenas aislados - Petróleo - minería - Madera - Perú - Ecuador.

\section{Abstract}

In Ecuador and Peru the contact between isolated indigenous groups and outsiders have increased. At LEAST IN THE RECENT PAST, THESE CONTACTS HAVE OCCURRED AS A RESULT OF OIL EXPLORATION THAT WAS CARRIED out in many areas in the Amazon Region. HoweVer, IT IS A Mistake to CONSIDER the COMPANIES AS PRINCIPAL protagonists of the problems that haVe arisen in many areas of the Amazon Basin. ON the contraRy, WE WILl ANALYZE THE ISSUE OF CONTACT FROM THE PERSPECTIVE OF THE "RESOURCE CURSE", THAT IS TO SAY, AS A CONSEQUENCE OF A SYSTEM CREATED BY NATIONAL ELITES TO APPROPRIATE THE OIL WEALTH "WITHOUT THE CITIZEN CONSENT". IN THIS SYSTEM THE OIL COMPANIES -PRIVATE OR PUBLIC- WORK AS AGENTS OR ACCOMPLICES

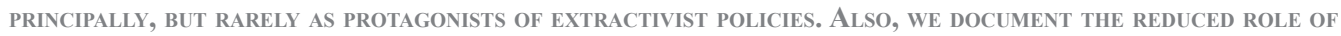
THE OIL INDUSTRY IN THE ISOLATEd GROUPS' TERRITORY, ESPECIALly IN PERU WHERE THIS GROUPS HAVE TO FACE GRAVE DANGERS SUCH AS THE CRIMINAL LOGGING, COCA AND GOLD BUSINESS.

KeyWORds: IsOleted INDIGENOUS GROUPS - OIL INDUSTRY - MiNING - LOGGING - PeRU - ECUADOR.

* Gerente General (jubilado) de Terra Group, Hershey, Pennsylvania, EE. UU. Doctor en Antropología Social de Harvard University, Cambridge, Massachusetts. Fue profesor de Antropología y Salud publica en Columbia University, Nueva York. Después, trabajó 40 años en la industria energética. Correo electrónico: wasserstromrobert@gmail.com. 


\section{Introducción}

$\mathrm{E}$ n la Región Amazónica, sobre todo en Ecuador y Perú, se aumentan los contactos entre grupos indígenas aislados y gente de afuera. A menudo, estos contactos se atribuyen a las actividades de empresas petroleras extranjeras, a las que acusan de hacer caso omiso de los derechos territoriales nativos'. Aquí abordamos el tema desde otra perspectiva: la relación entre la industria petrolera y las elites políticas nacionales que utilizan sus "privilegios sociales, políticos y jurídicos" (en las palabras del venezolano Juan Pablo Pérez Alonzo, cofundador de la OPEP) para apropiarse de la riqueza petrolera. No enfocamos a las empresas como protagonistas principales de las consecuencias nefastas ocurridas en muchas zonas de la cuenca amazónica. Al contrario, problematizamos las consecuencias de la "maldición de la abundancia" o "maldición de los recursos", es decir, el uso corrupto de los ingresos petroleros que permite a las elites gobernar "sin el consentimiento de los ciudadanos" (Karl, 1997; Soares de Oliveira, 2007). Evidentemente, a estas elites poco les interesa la suerte de los pueblos aislados.

Independientemente de que sean privadas o públicas (Petrobras, Rosneft o Sonangol), las empresas petroleras funcionan sobre todo como agentes o cómplices, no como dirigentes. Para entender su presencia en la Amazonía, también examinamos la estructura de la industria y sobre todo cómo éstas han venido evolucionando en los últimos 20 años. Tomamos como ejemplo principal el caso del Perú, donde actualmente viven 15 grupos aislados reconocidos, con algunas referencias al Ecuador. Y finalmente, evaluamos el reducido papel de las empresas petroleras como eje de penetración en los territorios de pueblos aislados. Según los datos resumidos aquí, estos pueblos ahora enfrentan un peligro mucho más grave: el negocio criminal de la madera, la coca y el oro.

\section{EI derecho al aislamiento y la responsabilidad estatal}

1995 fue el anno horribilis para la Royal Dutch/Shell Corporation, en ese entonces la segunda empresa petrolera privada del mundo ${ }^{2}$. El 10 de noviembre, el dictador nigeriano General Sani Abacha mandó ahorcar al famoso escritor Ken Saro-Wiwa, líder de una importante minoría étnica que se oponía a las operaciones de Shell en la Delta de río Niger. Shell insistió en que no había participado en la decisión del dictador, pero no le valió nada ante la furiosa reacción internacional. Y justo en ese momento, la compañía publicó sus planes para retirar de servicio una vieja boya de almacenamiento en el mar del Norte llamada Brent Spar. Según Shell, existía menos riesgo de contaminación si se hundía en las aguas profundas del mar que si se remolcara a algún puerto para desmontarla. El público europeo no se tranquilizó con esta explicación. Durante tres semanas, la Brent Spar fue ocupada por Greenpeace; en Europa, un boicot de las gasolineras de Shell duró varios años más.

En ese contexto Shell formuló su política de pueblos aislados en Perú. Desde 1984, había buscado hidrocarburos en la selva amazónica, donde hizo un enorme descubrimiento de gas natural en Camisea. El gobierno de Alan García, dueño del gas, quería acelerar el desarrollo del yacimiento que aportaría US \$34.000 millones al fisco (Hydrocarbons-technology.com, 2013). Sin embargo, dentro de Shell, hubo cautela. El campo de Camisea colindaba con el Parque Nacional Manú, reconocido por la UNESCO como Patrimonio de la Humanidad y Reserva de la Biosfera. Estaba ubicado en el Departamento de Madre de Dios, una región de bosque tropical que seguramente llamaría la atención de las organizaciones internacionales. De hecho, ya habían surgido algunos problemas: en 1984, algunos trabajadores de la compañía encontraron a un grupo de indígenas nahua, quienes pronto se enfermaron. Para evitar mayores

1 Para una discusión de esta "narrativa estándar", ver Reider y Wasserstrom (2013).

2 En este artículo, "petróleo" se refiere globalmente a todos los productos hidrocarburíferos: petróleo, gas natural, etc. 
escándalos, en 1990 el gobierno peruano delineó una Reserva Territorial Kugapakori-NahuaNanti "para los pueblos no contactados o en aislamiento voluntario" (Feather, 2014: 5). Pero nunca renunció a sus derechos petroleros dentro de las reservas nativas. En cualquier momento, los podía delegar a Shell o a otro contratista.

La política de Shell incorporó una serie de medidas para evitar el contacto con pueblos aislados y minimizar las posibilidades de contagiarlos si el contacto ocurriera (Zarzar, 2000: 60). En la imaginación popular, estos grupos representaban una herencia casi bíblica: los últimos sobrevivientes de la Edad de Piedra. Sin embargo, era poco probable que nunca hubiesen tenido tratos con el mundo exterior. Al contrario, casi seguramente habían rechazado el contacto destructivo iniciado durante la época cauchera (1885-1930) y seguido durante la expansión agrícola comercial subsecuente (Huertas, 2004: 20). Como toda comunidad indígena en Perú, los grupos en aislamiento voluntario tenían derecho (reconocido desde 1989 por la Organización Internacional de Trabajo en su Convenio 169) a la consulta previa antes de comenzar cualquier actividad económica en su territorio ${ }^{3}$. ¿Pero cómo se inicia un diálogo que los grupos mismos habían rechazado? ¿Y en qué forma podrían participar realmente en las discusiones oficiales? (Feather, 2014: 4).

También existía un obstáculo adicional. Según el Convenio 169 y otros acuerdos internacionales, la consulta previa - como los demás derechos humanos y civiles - era una responsabilidad principal de los gobiernos, no de las empresas petroleras. A pesar de ello, los gobiernos con frecuencia preferían delegar estas obligaciones a sus contratistas petroleras o mineras. En 1984 o 1985, por ejemplo, Petroecuador implementó una política que luego se extendió a casi toda la región amazónica: los contratistas privados deberían negociar un "acuerdo comunitario" con las comunidades directamente impactadas por sus actividades (Reider y Wasserstrom, 2013). Como los gobiernos raramente intervenían o ponían normas, de pronto surgieron nuevas formas de desigualdad entre comunidades que recibían tratos distintos. En vez de ser un mecanismo de justicia, los "acuerdos comunitarios" rápidamente se convirtieron en una nueva fuente de conflictos. Y hasta las compañías mejor intencionadas se enfrentaban con el espectro de los contadores estatales que podían vetar cualquier inversión comunitaria como innecesaria o demasiado generosa.

En 1996, se organizó bajo el auspicio de la universidad de Harvard una mesa de trabajo para elaborar - aun informalmente - un código de conducta para las empresas petroleras en la región amazónica. Participaron todas las compañías con operaciones importantes, más las principales organizaciones indígenas y ambientales. Las empresas estatales se negaron a asomarse porque, según ellas, eran soberanas y no tenían ninguna obligación de consultar a sus ciudadanos o a grupos particulares. Los "Diálogos de Harvard" duraron tres años, pero se abandonaron porque a la final nadie sabía si los gobiernos aceptarían el condigo de conducta.

En 2008, el papel protagónico del estado frente a las comunidades indígenas fue enfatizado por el Consejo de Derechos Humanos de las Naciones Unidas (UNHRC en inglés). En un documento conocido como el "Marco Ruggie", se afirmó la responsabilidad exclusiva del Estado de promulgar leyes, normas, reglas y otras medidas que protejan los derechos de sus ciudadanos (UNHRC, 2011)4. Según el documento, las compañías privadas también deben respetar estos derechos, incluso cuando las normas jurídicas locales o nacionales sean inadecuadas para garantizar su aplicación. Pero su papel siempre es complementario:

3 Estos derechos fueron reafirmados también en la "Declaración de las Naciones Unidas sobre los derechos de los pueblos indígenas" (2007) y el "Decreto Supremo No. 012-2008-EM" (Presidente Constitucional de la Republica, 2008). Una crónica de la legislación peruana relevante se encuentra en Consulta (2015).

4 El título completo del documento es "Promoción y protección de los derechos humanos, civiles, políticos, económicos, sociales y culturales, incluyendo el derecho al desarrollo. Proteger, respetar y remediar: un marco de los derechos humanos para las empresas privadas" (traducción nuestra). Para los pueblos en aislamiento, ver también ACNUDH (2012). 


\begin{abstract}
"Las empresas no pueden ser consideradas como responsables de los impactos ejercidos por cualesquiera de las entidades sobre las cuales tienen alguna influencia... sobre todo, los gobiernos... Pidiendo a las empresas que apoyen voluntariamente los derechos humanos donde tengan alguna influencia es una cosa, pero adjudicándoles la responsabilidad principal es un asunto muy distinto... Un gobierno podría intencionalmente negarse a cumplir con sus propias obligaciones conservando la esperanza o expectativa de que la empresa ceda a las presiones sociales para promover o afirmar estos derechos" (UNHRC, 2008: 12-20; traducción nuestra)".
\end{abstract}

Con esto, el autor principal del documento, el profesor John Ruggie de la Escuela de Derecho de Harvard, ponía en tela de juicio un argumento común - sobre todo entre la comunidad activista - que traspasaría a las contratistas privadas ciertas obligaciones básicas del Estado, mientras cerraban los ojos ante la evidente incapacidad, indiferencia o corrupción de los gobiernos.

\title{
Hidrocarburos y reservas fantasmas
}

De hecho, Ruggie criticaba una falla importante en la estrategia de pasar por alto la conducta oficial para enfocarse casi exclusivamente en las actividades de las empresas. En vez de salvaguardar a los pueblos aislados, esta estrategia de "campañas ambientales permanentes" aumentaba su vulnerabilidad. Según el Banco Mundial, todas las empresas petroleras privadas juntas ahora controlan tan sólo el 10\% de los recursos hidrocarburíferos globales, mientras el $90 \%$ está en manos de los Estados. Las compañías grandes - las que son más sensibles a la opinión pública - ya operan principalmente donde vive poca gente: el mar profundo, Siberia, el Ártico. Esto obedece a la lógica del mercado internacional. El valor de una empresa petrolera no está determinado por sus ventas o sus ingresos, sino que depende mayormente de las reservas probadas que pueda explotar ${ }^{5}$. En este momento, por ejemplo, ExxonMobil posee 25,3 mil millones de barriles de reservas probadas en todo el mundo; a un precio hipotético de US $\$ 50$ por barril, sus reservas representarían un valor total de US\$1.165 mil millones. Pero Exxon también vende 1,4 mil millones de barriles por año - una proporción apreciable de sus reservas totales. Si no los reemplazara, Exxon desaparecería en 18 años.

Entonces, ¿dónde encuentra los enormes nuevos descubrimientos - los llamados "campos elefantes" - que necesita Exxon para sobrevivir? ¿Y dónde existen las condiciones geológicas, técnicas o logísticas tan difíciles que las empresas estatales - dueñas de casi todos los campos elefantes del mundo - no puedan explotarlos sin la ayuda de Exxon? Sobre todo en el golfo de México, el golfo de Guinea (África Occidental) y el extremo norte de Rusia. Efectivamente, allí se concentran las grandes compañías privadas con su avanzada tecnología y su alta capacidad económica de enfrentar riesgos extraordinarios. Mientras tanto, en las zonas convencionales como la Amazonía, predominan las empresas medianas y pequeñas o incluso algunas estatales nacionales y extranjeras como Petrochina que aceptan menos riesgo a cambio de menos remuneración.

En Perú, por ejemplo, el único descubrimiento grande hasta ahora fue Camisea. Ante el fracaso de negociar un contrato de producción satisfactorio, Shell salió del país en 1998. La sustituyó un consorcio encabezado por Pluspetrol, una compañía argentina mediana no sólo privada sino particular. Pluspetrol no vende acciones en las bolsas de Nueva York o Londres y por ende no está sujeta al escrutinio de las agencias reguladoras internacionales. Proporciona muy pocos datos al público. En Camisea, el gobierno peruano designó a Pluspetrol como "operadora," accionista con el $27,2 \%$ del consorcio y con la responsabilidad exclusiva de producir el gas. Los demás accionistas - Hunt Oil (EE. UU., 25,2\%), SK Energy (Corea del Sur, 17,6\%), Tecpetrol (Argentina, 10\%), Repsol (España y Argentina, 10\%) y Sonantrach

5 Las reservas probadas normalmente se calculan a base de los barriles de petróleo (o su equivalente en gas natural) que puedan producirse con un $90 \%$ de probabilidad en los próximos cinco años utilizando la tecnología existente. 
(estatal de Argelia, 10\%) - son inversionistas pasivos que reciben una proporción de los ingresos de acuerdo a su aportación financiera (TOGY, 2015). Fuera de Camisea, Pluspetrol también opera varios proyectos y concesiones peruanas. No se sabe realmente con quién más estaría asociada.

En 2000, después de otorgarle el contrato de Camisea a Pluspetrol, el gobierno incorporó la zona gasera en una nueva concesión, llamada Lote 88. Pero a diferencia del Camisea anterior, el Lote 88 también abarcaba el $23 \%$ de la reserva territorial con sus ocho grupos en aislamiento voluntario (Feather, 2014: 6). En 2003, cambió el estatus jurídico de la reserva nativa KugapakriNanti colindante a Camisea para transformarla en "Reserva Territorial del Estado", donde supuestamente se prohibía cualquier asentamiento o actividad económica (Barclay y García Hierro, 2014). Sin embargo, con el visto bueno del gobierno, Pluspetrol inició casi de inmediato un programa de exploración geológica en las zonas protegidas. En 2012, pidió permiso para perforar 18 pozos adicionales y construir una línea de flujo atravesando la zona de exclusión económica. La propuesta fue aprobada sin demora - a pesar de la intensa oposición de grupos indígenas, ambientalistas y hasta los mismos expertos oficiales (Ministerio de Cultura, 2013; Hill, 2013). El Viceministro de Interculturalidad (encargado de asuntos indígenas) dio una explicación salomónica: “el Lote 88 se trazó en el año 2000, antes del 2003, fecha de la creación de la reserva Nahua-Kupakagori por decreto supremo" (Collyns, 2013). O sea que el gobierno no vaciló en desconocer los límites de la reserva indígena que había decretado en 1990 porque ya estorbaban sus ambiciones de ampliar la zona productiva. En seguida, autorizó un extensivo programa de trabajos exploratorios fuera del Lote 88 en el Parque Nacional Manú (Hill, 2015). Actualmente, como lo notan Barclay y García Hierro (2014: 38), se contempla extinguir la reserva nativa territorial por completo ${ }^{6}$.

Estas circunstancias no se limitan ni a Camisea ni a Pluspetrol (Huertas, 2010; 2013). En 2006, el gobierno peruano adoptó una política de pueblos aislados semejante a la brasileña, vigente desde 1986. En vez de atraerlos a la sociedad moderna, como antes, esta política reconocía la inviolabilidad de sus territorios y trataba de mantenerlos a salvo de las epidemias y otros impactos del contacto. Al año siguiente, empero, el presidente de Perúpetro (la empresa estatal) declaró que "es absurdo afirmar que existan pueblos no contactados" (Survival International, 2007a). Su opinión fue reafirmada poco después por el Presidente Alan García, quien declaró que los grupos aislados en el país eran un invento ambientalista para mermar el progreso de los demás peruanos "como el perro del hortelano, que no come ni deja comer" (Survival International, 2007b; Carroll, 2009). Pronto se conocieron las dimensiones del jardín. Se trataba del Lote 67, ubicado entre los ríos Napo y Tigre cerca de la frontera con Ecuador. En 2006, Barrett Resources (bajo contrato con Perúpetro) hizo un descubrimiento petrolero estimado en 300 millones de barriles (El Comercio, 2013; Ríos Villacorta, 2014) En 2011, la operadora del proyecto, una empresa francesa llamada Perenco, pidió permiso para construir una nueva línea de flujo de $200 \mathrm{~km}$. en un área posiblemente frecuentada por grupos aislados. Según el periodista David Hill (2011), la presencia de los "no contactados", planteada desde antes por Barrett en sus estudios de impacto ambiental, fue totalmente omitida en el EIA de Perenco. "Perenco tuvo todo lo que quería", declaró uno de los expertos que preparó el documento. Pero aquí cayó en una trampa ideológica. ¿Acaso no fue Perúpetro que tuvo lo que quería: una inversión de $\$ 1.800$ millones que conectaba la zona fronteriza - sin explotar por falta de transporte - al Oleoducto Norperuano? Si el EIA o el plan de manejo eran deficientes, ¿a quién le correspondía exigir que se corrigieran? Perenco era contratista, no autoridad: carecía de toda facultad que Perúpetro no le concediera.

6 El gobierno ecuatoriano hizo algo parecido cuando sustrajo una parte del Parque Nacional Yasuní, donde la actividad petrolera estaba prohibida, para agregarla al territorio étnico waorani donde estaba permitida.

7 Los contratos celebrados por Perúpetro se pueden consultar en: http://www.perupetro.com.pe/relaciondecontratos/ relacion.jsp?token $=106$. 


\title{
Madera, minería y coca
}

La crisis enfrentada por los pueblos aislados en el Lote 67 y en Camisea no se explica en función de la codicia de una sola empresa ni siquiera del comportamiento colectivo de la industria petrolera en su totalidad. Es el resultado de una política nacional de desarrollo en la Amazonía peruana que pasa por alto los costos para las poblaciones indígenas. Según Huertas (2010: 6; ver también Huertas, 2013; Marris, 2016), estos grupos

\begin{abstract}
"son una de las expresiones más contundentes de rechazo a una historia de injusticia social, despojo territorial, desaparición física y cultural ocasionada tanto por acción como por omisión del Estado. Por acción, a través de la imposición de un modelo de desarrollo económico de data colonial, consistente en la puesta en valor de sus tierras y recursos naturales y el fomento de las inversiones para su explotación, sin tener en cuenta que estos son condición fundamental para su existencia. Por omisión, ante...la violación continua de sus derechos fundamentales de parte de agentes externos que los despojan de sus territorios, fuerzan contactos o se enfrentan violentamente a ellos, con diferentes fines".
\end{abstract}

En Madre de Dios, esta situación se remonta a finales del Siglo XIX. Los Nahua de Camisea - fragmentos de un grupo más amplio conocido también como Mashco Piro - eran en realidad sobrevivientes del imperio cauchero de Carlos Fermín Fitzcarrald. A finales del siglo XIX, Fitzcarrald "rastreó la selva en busca de indígenas asháninka y piro que fueron obligados a trabajar en sus concesiones en los ríos Urubamba, Manú y Mishagua" (Brown y Fernández, 1991: 63; traducción nuestra). Con el colapso definitivo del boom cauchero en 1930, algunos Mashco Piro se adentraron en la selva para transformarse en grupos "no contactados" (Gow, 1991).

Sin embargo, su tiempo de aislamiento fue limitado. En 1940, los misioneros dominicos de Puerto Maldonado entraron a su territorio para "reducirlos" a la vida civilizada. Con ello, desataron una epidemia que cobró no se sabe cuántas vidas (Fernández Moro, 1952: 649). Ya en los años 50s, como lo describe Beatriz Huertas (2012: 36),

\footnotetext{
“el gobierno peruano reinició la construcción de carreteras y aeródromos con lo que las migraciones a Madre de Dios fueron incrementando. Poco después se inició un ciclo de extracción de castaña y, en la década de 1960, cuando llegó la carretera de Cusco, se intensificó la extracción forestal. A lo largo de la década de 1970, el auge de la minería, la construcción de la carretera Cusco-Shintuya y las políticas de colonización promovidas por el gobierno genera el ingreso de grandes contingentes de migrantes. Durante las décadas de 1970 y 1980, el oro asume el papel predominante en la economía de Madre de Dios y fortalece a los grupos de poder regional. A la vez se mantienen las actividades de extracción de madera y castaña".
}

Con los migrantes, sobre todo los madereros, llegaron las enfermedades: en 1986, por ejemplo, murió hasta el 60\% del grupo nahua (Survival International, s/f). "Había tantos cuerpos que no los alcanzamos sepultar", comentó uno de los sobrevivientes. "Los comieron los gallinazos" (Carrington, 2014).

Actualmente, la amenaza más grave que enfrentan los grupos aislados no es principalmente el petróleo, sino la altamente organizada tala ilegal de madera. Como lo explica Huertas (2004: 66; traducción nuestra),

"las especies de alto valor comercial como la caoba y el cedro son escasas en grandes extensiones
de Madre de Dios porque fueron explotadas intensivamente desde los $1970 \mathrm{~s}$. Sin embargo,
todavía abundan en las zonas remotas ocupadas por los pueblos aislados. Esto ha generado un
movimiento masivo de empresas madereras y de extractores particulares hacia la zona, sobre
todo desde finales de los 1990s. En las últimas décadas, la explotación maderera ilegal ha pasado
por varias etapas distintas: el boom de los primeros años, envuelto en un escándalo internacional 
a causa de los altos volúmenes cortados y las redes de corrupción involucradas; un período de incertidumbre surgida de la aplicación de medidas jurídicas radicales adoptadas para parar ese comercio; un lapso corto de vedas y decomisos; y ahora una etapa de ajuste a la 'legalización' de la extracción ilegal con el uso irregular de una nueva documentación exigida por las autoridades correspondientes".

Según el Banco Mundial (Pereira et al., 2012), "La mayoría de las operaciones de deforestación de este tipo son realizadas por bandas de crimen organizado y las ganancias muchas veces acaban en los bolsillos de funcionarios corruptos".

En Perú, el $80 \%$ de la madera exportada se trafica ilegalmente (Ibíd.: 2). Además de comercializar los troncos cortados dentro de sus concesiones, las empresas emplean documentos falsos para exportar caoba y cedro sacados de las reservas nativas o áreas protegidas (Neuman y Zárate, 2013; Martins, 2014; Global Witness, 2014). “Desde el patrón que vende a aserraderos que a su vez venden a otros intermediarios, la madera se moviliza con papeles aparentemente legales pero que no reflejan la realidad", explica Martins. "Hay un sistema legal que funciona en paralelo. El sistema de concesiones forestales se usa para lavar madera". En un estudio de la Reserva Territorial Purús, por ejemplo, se contaron 127 campamentos ilegales con 1.110 madereros pequeños o trabajadores "habilitados" por las empresas grandes (Huertas, 2004: 71). El 31 de agosto de 2014, Edwin Chota, un líder Asháninka que se opuso al saqueo de su territorito, fue asesinado por los cabecillas de una pandilla local (Zárate y Neuman, 2014; Wallace, 2014; Global Witness, 2014) ${ }^{8}$. En 2015, agentes de la fiscalía ambiental y OSINFOR (el Organismo de Supervisión de los Recursos Forestales y de Fauna Silvestre, encargado de controlar la explotación maderera) lograron parar un cargamento equivalente a 60 camiones pesados destinado a EE. UU. y México (Luna Amancio, 2015). Pero el jefe de OSINFOR pagó caro esta indiscreción: la "mafia maderera" consiguió destituirlo de su cargo (Environmental Investigation Agency, 2016; Collyns, 2016).

A la explotación maderera hay que sumar dos amenazas más: la minería y la coca. La minería ilegal ocupa ahora 43.000 ha; mientras 8.000 ha de coca se cultivan en 13 áreas protegidas que abarcan los territorios de grupos en aislamiento (Finer y Novoa, 2015; Finer y Novoa y Olexy, 2016a; 2016b; 2016c) ${ }^{9}$. Con esto, se han vuelto más frecuentes los contactos inesperados entre grupos aislados y las comunidades indígenas cercanas. En el Alto Purús, por ejemplo, la "mafia de la caoba" mantiene una extensiva red de transporte vinculando las reservas indígenas con Brasil; esta red también sirve para exportar coca a Europa ${ }^{10}$. Hasta recién, comentan Salisbury y Fagan (2011), los grupos en aislamiento hubieran evitado el contacto con los intrusos, pero ahora no hay cómo porque sus territorios están salpicados por caminos ilegales. Los enfrentamientos violentos no se hicieron esperar. En 2014,

"uno de los grupos más aislados del mundo empezó a salir de la selva amazónica profunda y buscar el contacto con las comunidades asentadas. El contacto excepcional con este grupo, parte de la etnia Jaminawa, sugiere que los traficantes de drogas estén presionando al pueblo nómada para salir de sus tierras selváticas ya que la frontera entre Brasil y Perú se ha convertido en una ruta de distribución importante de la coca" (Watts, 2014; véanse también Pringle, 2014: 125; Macqueen, 2016).

8 El Caso Chota se volvió célebre, pero no es el único. También murió Mauro Pío, líder de una comunidad indígena "superpuesta a una concesión forestal de la empresa Bailarín" (Fawks, 2014). "No se ha conocido responsables, no hubo interés del Estado en hacer una investigación", comentó la presidenta de una organización asháninka.

9 La minería en Madre de Dios y sus impactos en poblaciones indígenas durante el Siglo XX también se describen en Moore (2003) y Urteaga (2003). A principios de 2016, ha logrado incluso alterar el curso del río Malinowski y los límites de la Reserva Nacional Tambopata (Finer y Novoa, 2016).

10 Grillo (2016: 81) describe los mecanismos de exportación de la coca peruana y colombiana desde Brasil hacia Europa. 
Otro impacto dramático de estos negocios ilícitos se manifiesta en lo que Ferguson y Whitehead (1992) llaman "la guerra en la zona tribal". En esa zona, el Estado lejano suscita conflictos, alianzas y competencias entre grupos étnicos que se hacen y deshacen según las presiones ejercidas desde "más allá de la frontera". Esta descripción aclara lo que pasa actualmente en Madre de Dios y Ucayali, donde vive la mayoría de los 15 pueblos aislados en el Perú. El 28 de octubre de 2008, por ejemplo, "un niño indígena de catorce años ha sido herido de gravedad en lo que se cree ha sido un ataque de indígenas [Mashco Piro] cerca de su casa en Monte Salvado" (Survival International, 2010). Monte Salvado es una comunidad yine ubicada en el río Las Piedras, importante ruta de tránsito entre los bosques protegidos y la reserva territorial indígena. Su idioma es parecido al de los Mashco Piro, con los que tenían contactos esporádicos durante dos generaciones. En junio de 2013, aparecieron más de 100 Mashco Piro en la ribera opuesta a Monte Salvado para exigir alimentos, cuerdas y machetes (Associated Press, 2013).

Al año siguiente, regresaron 200 Mashco con arcos y flechas y saquearon al pueblo. Rompieron cristales, mataron animales domésticos, destrozaron la ropa que encontraron en las casas; se llevaron machetes, comida, cuerdas y cobijas. La comunidad quedó evacuada por algún tiempo (Collyns, 2014; FENAMAD, 2014). Entre 2014 y 2015, los Mashco Piro aparecieron más de 100 veces en las riberas del río Las Piedras para pedir regalos a los turistas y algún misionero (Lawler, 2015a: 679). En 2015, un residente de Monte Salvado fue alcanzado por una flecha. "Definitivamente, uno ve como la frecuencia y agresividad de los contactos están acelerando", observa el antropólogo Glenn Shepard (en Wallace, 2015). Durante 2015 y 2016, hubo múltiples incidentes semejantes a lo largo de la zona fronteriza (Lawler, 2015b). Comenta un líder indígena: "Nuestra teoría es que los hermanos no contactados de la Reserva Territorial están buscando nuevas tierras en las que vivir".

En Ecuador, la masacre de los Taromenani en 2013 por sus vecinos Waorani tiene orígenes semejantes. Según Cabodevilla (2007: 17), los 100 Taromenani vivían en una zona abandonada cerca del río Curaray y la frontera peruana que no les disputaba nadie. Al contrario, los Waorani prefirieron agruparse a lo largo del camino petrolero construido en 1972, la llamada vía Auca (Wasserstrom, 2016). Igual que en Perú, este camino ofrecía acceso a los madereros y colonos agrícolas que querían explotar la reserva étnica waorani. Para algunas familias waorani en la vía Auca, la migración era negocio: cobraban para dejar pasar los troncos cortados en la reserva y en la zona de amortiguamiento del Parque Nacional Yasuní. Con el tiempo, se agotó la madera preciosa cercana y los madereros empezaron a penetrar en los territorios más lejanos. Allí encontraron una resistencia tenaz de los Taromenani. Hubo muertos. En 2013, algunos waorani decidieron acabar con el problema que amenazaba su negocio. Asaltó a las malocas taromenani y destruyeron a sus habitantes (De Marchi, Aguirre y Cabodevilla, 2013). Las autoridades apenas intervinieron. En 2014, la Comisión Interamericana de Derechos Humanos (CIDA) declaró que el gobierno ecuatoriano había incurrido en la violación de los derechos humanos taromenani por no haber tomado medidas efectivas de protección - a pesar de un dictamen de CIDA en 2006 (CIDA, 2014; ver también Corte Interamericana, 2014). De la prensa y algunas ONGs se emitió una densa nube de mitificación sobre las supuestas tradiciones indígenas de guerra y venganza ${ }^{11}$. Pero en realidad fue la crónica de una muerte anunciada desde mucho antes en la "zona tribal".

11 Ver, por ejemplo, el comentario de Colleoni y Proaño (2010: 9; 2013) sobre el "espiral de venganzas, raptos y violencias" entre los dos grupos. A nuestro juicio, esta forma de esencialismo le da una cobertura al Estado para no proteger a los grupos vulnerables bajo el pretexto de respetar sus culturas. Ignora el contacto episódico - aunque no siempre pacífico - entre los grupos waorani y taromenani durante un período evidentemente prolongado. Después de la masacre en 2003, por ejemplo, se encontraron hachas de acero, machetes, ollas, etc., en las malocas taromenami destruidas. ¿De dónde habrán salido esos objetos? También es muy posible que algunos Taromenani hayan salido de vez en cuando a trabajar en los campos petroleros, como lo hicieron los Tetete (otro grupo desaparecido) con los caucheros a principios del Siglo XX. 


\section{La maldición de la abundancia}

Dentro de este panorama complicado, ¿cuál es el papel de las empresas petroleras privadas? En ambos países, los caminos petroleros facilitaban la invasión inicial de madereros, productores de coca, mineros ilegales, etc. Desde el punto de visto técnico, empero, ya se sabe cómo minimizar este problema. En todas las regiones del mundo, las plataformas marítimas se construyen sin caminos: se utilizan helicópteros y barcos. Este modelo "costa afuera" o "mar adentro" se aplicó con éxito hace 20 años en Ecuador, en el Proyecto Villano (Bloque 10); costó sólo el 10\% más que los métodos de construcción tradicionales ${ }^{12}$. Algunos funcionarios seguramente argumentarían que ese $10 \%$ se gasta a costo de los demás ecuatorianos o peruanos, porque de otra forma se invertiría en salud, educación, infraestructura y otros beneficios. A nuestro juicio, este argumento queda sin mucho peso: ambos países se han comprometido a salvaguardar los derechos de los pueblos aislados y su compromiso implica algún costo. El $10 \%$ de los gastos de construcción no parece un precio exorbitante.

Entonces, ¿por qué se siguen construyendo caminos de acceso en las zonas protegidas? Volvamos a "la maldición de los recursos". Este concepto se originó con el economista inglés Richard Auty (1993: 1; traducción nuestra), quien analizó el sector minero en Perú y Chile. Auty notó una paradoja importante:

"La visión normal del papel de los recursos naturales en el desarrollo económico es que los recursos son más críticos en las etapas iniciales del proceso... [Sin embargo,] muchos países ricos en recursos no experimentan un impacto positivo, sino que tienen resultados inferiores a los países menos dotados".

Este modelo fue aplicado a la industria petrolera por Terri Lynn Karl, una politóloga especialista en Venezuela. Karl (1997: xvi; traducción nuestra) se enfocó en las distorsiones políticas y económicas impuestas por las élites que dominan la explotación de recursos naturales:

"El marco para tomar decisiones, o sea, los incentivos dentro de una economía política determinada... reflejan y son producto de las relaciones de poder, actuales o anticipadas...Tienden a persistir...a pesar de que pueda existir un reconocimiento generalizado de que son mal adaptados o que deban alterarse".

En estas circunstancias, la corrupción no es un simple resultado inesperado del sistema político ineficiente o incapaz, sino "la forma normal en que opera el estado" (Soares de Oliveira, 2007: 138). Como lo observa Ricardo Soares de Oliveira (2007: 53; traducción nuestra),

“...ninguna explotación petrolera se llevaría a cabo sin una asociación estrecha entre el estado y las empresas. Esto quiere decir que las empresas no simplemente evitan estorbar al estado; [sino] que refuerzan su poder..., ayudan a obtener los préstamos que [el estado] no podría conseguir con sus propios esfuerzos y, de mayor importancia, le dan acceso a las ganancias de un recurso que de otra forma quedaría fuera de su alcance".

Los problemas técnicos muy probablemente podrían superarse, pero los pueblos aislados siempre se quedarán vulnerables ante un Estado que los ve como irrelevantes. Observa Huertas (2010: 1), "este comportamiento del Estado no es casual; por el contrario, está orientado a

12 En la Amazonia occidental, los impactos negativos de la explotación petrolera casi siempre están asociados con la construcción de caminos (Finer et al., 2008). Estos impactos igualmente ocurren en zonas donde hay caminos pero no existe petróleo (Santos Granero y Barclay, 1998; Wasserstrom y Southgate, 2013). Evidentemente, la solución cuando menos parcial - es evitar o controlar el acceso a las zonas protegidas. 
fortalecer la política económica en base a la flexibilización y debilitamiento de la normatividad e institucionalidad que debería amparar los derechos de los pueblos indígenas, facilitando así la disponibilidad de sus territorios para la inversión"13.

Ahora este problema se está volviendo todavía más complicado. En Perú y sobre todo en Ecuador, los gobiernos están concentrando la exploración petrolera en manos de dos empresas chinas estatales, la Chinese National Petroleum Corporation (CNPC) y Sinopec. China es la mayor importadora de hidrocarburos del mundo, mientras Ecuador produce una cantidad mínima: alrededor del 0,5\%. ¿Por qué le interesa a China operar en Ecuador? El modelo económico de China se basa en la constante inversión estatal o paraestatal, sobre todo en grandes obras de construcción e infraestructura. Pero el mercado chino está saturado: no existen buenas posibilidades de invertir en proyectos que alcancen las altas tasas de rendimiento acostumbradas (Pozzebon, 2015). En África y América Latina, el Estado chino y sus capitalistas afiliados siguen un doble objetivo: invierten en los sectores extractivos $a$ cambio de construir represas, ferrocarriles, puertos, caminos, minas, refinerías, etc. (Downs y Evans, 2006; Sanderson y Forsythe, 2012). Por lo general, estos proyectos proporcionan atractivos rendimientos muy por encima de lo que se podrían obtener en la decaída industria petrolera (Aisch, Keller y Lai, 2015). A diferencia del Banco Mundial y otras fuentes de financiamiento convencionales, tampoco ponen difíciles requisitos ambientales o sociales. Están dispuestos a trabajar "sin meterse en política", como declara erróneamente la prensa financiera internacional. En la práctica, esto implica una postura política muy intervencionista: reforzar el poder de las elites existentes en países autoritarios haciéndoles socios de su programa económico (Wasserstrom, 2015).

¿Cómo responder a estos desafíos? Evidentemente, la respuesta tradicional - organizar campañas de opinión pública contra tal o cual empresa privada - ya dará resultados muy pobres. De hecho, estamos ante un nuevo boom amazónico, pero con una diferencia crítica: el Estado mismo - y las elites políticas que lo dominan - se beneficia directamente del saqueo. Para los pueblos vulnerables, terminará como el primer boom si no logramos frenar la maldición de esta abundancia. Los cambios que se requieren son profundos, no de "responsabilidad social corporativa". Implica una nueva estrategia política que ni siquiera se ha imaginado. Pero es la única opción que nos queda. Lo demás ya fracasó.

13 También lo enfatiza la Comisionada de Derechos Humanos de la OEA Dinah Shelton (201: 1; traducción nuestra): "Es notable el grado de convergencia entre los pueblos aislados del continente, no obstante las fronteras internacionales actuales. Los desafíos y peligros se asemejan: la invasión lenta pero persistente de sus territorios, la explotación legal e ilegal de sus recursos naturales... Mientras tanto, los gobiernos tienden a reaccionar en una forma semejante: el reconocimiento mínimo de su existencia y sus derechos, junto con la notable falta de intervención en la práctica". 


\section{Bibliografía}

Aisch, Gregory, Josh Keller y K. K. Rebecca Lai. 2015, "The World According to China”, New York Times, 4 julio 2015, http://www.nytimes.com/interactive/2015/07/24/business/international/the-world-according-tochina-investment-maps.html?_r=0

Alto Comisionado de las Naciones Unidas para los Derechos Humanos (ACNUDH). 2012, "Directrices de Protección para los Pueblos Indígenas en Aislamiento y en Contacto Inicial de la Región Amazónica, el Gran Chaco y la región oriental de Paraguay". Ginebra: ADNUCH.

Associated Press (Lima). 2013, "Peru: alarm over appearance of isolated Mashco-Piro tribe", 20 August 2013, http://www.theguardian.com/world/2013/aug/20/peru-appearance-isolated-mascho-piro-tribe.

Auty, Richard. 1993, Sustaining Development in Mineral Economies. The resource curse thesis, Routledge, Londres y Nueva York.

Barclay, Frederica y Pedro García Hierro. 2014, La batalla por los "Nanti”, Perú Equidad, Lima.

Brown, Michael F. y Eduardo Fernández. 2011, War of Shadows. The Struggle for Utopia in the Peruvian Amazon, University of California Press, Berkeley.

Cabodevilla, Miguel Ángel. 2007, El exterminio de los pueblos ocultos, Centro de Investigación Cultural de la Amazonía Ecuatoriana (CICAME), Quito.

Carrington, Damian. 2014, "Amazon tribe makes first contact with outside world", The Guardian, 1 agosto 2014, http:/www.theguardian.com/world/2014/aug/01/amazon-tribe-makes-first-contact-with-outsideworld.

Carroll, Rory. 2009, "Rumble in the jungle", The Guardian, 3 julio 2009, http://www.theguardian.com/ environment/2009/jul/04/peru-amazon-rainforest-conservation?INTCMP=SRCH.

Colleoni, Paola y José Proaño. 2010, Caminantes de la selva. Los pueblos en aislamiento de la Amazonía Ecuatoriana, Informe 7, International Work Group on Indigenous Affairs (IWGIA), Copenhague.

Colleoni, Paola y José Proaño. 2013. "Isolated Peoples of the Ecuadorian Amazon”, en: Dany R. Mahecha y Carlos y Eduardo Franky C., (Eds.) Indigenous Peoples in Voluntary Isolation and Initial Contact, International Working Group on Indigenous Affairs (IWGIA), Copenhague, pp. 158-199.

Collyns, Dan. 2016. "Peru sacks top anti-logging official", The Guardian, 19 enero 2016, http://www.theguardian. com/environment/2016/jan/19/peru-sacks-top-anti-logging-official-rolando-navarro.

2014. "Peru evacuates Amazon village after raid by indigenous tribe", The Guardian, 23 diciembre 2014, http:// www.theguardian.com/world/2014/dec/23/peru-evacuates-village-tribal-raid.

2013. "Los pueblos indígenas de Perú recurren a los tribunales contra la exploración de gas", The Guardian, 3 enero 2013, http:/www.theguardian.com/global-development/2013/jan/03/peru-indigenas-exploracion-gas.

Comisión Interamericana de Derechos Humanos (CIDH). 2014, "Informe de Admisibilidad. Pueblos Indígenas en Aislamiento Tagaeri y Taromenani. Ecuador”, Informe No. 96/14. Petición 422-06, Organización de los Estados Americanos, Washington.

Consulta. 2015. "Legislación," http://consultape.com/legislacion/.

Corte Interamericana de Derechos Humanos. 2014. "Resolución de la Corte Interamericana de Derechos Humanos de 31 de marzo de 2014. Medidas provisionales respecto de Ecuador. Asunto respecto a dos niñas del pueblo indígena taromenane en aislamiento voluntario", San José de Costa Rica: Corte Interamericana de Derechos Humanos.

De Marchi, Massimo, Milagros Aguirre y Miguel Ángel Cabodevilla. 2013, Una tragedia ocultada, Fundación Alejandro Labaka, Quito.

Downs, Erica y Peter Evans. 2006, "Untangling China’s Quest for Oil through State-backed Financial Deals", Washington, Brookings Institution, http:/www.brookings.edu/research/papers/2006/05/china-downs.

El Comercio (Lima). 2013, "Perenco inició la producción de crudo pesado en el Lote 67 en Loreto". 4 diciembre 2013, http://elcomercio.pe/economia/negocios/lote-67-perenco-inicio-produccion-crudo-pesado-loretonoticia-1668525.

Environmental Investigation Agency. 2016, "EIA Denounces Termination of Peruvian Forest Oversight Body President”. Washington, EIA, http://eia-global.org/news-media/eia-denounces-termination-of-peruvianforest-oversight-body-president.

Fawks, Jacqueline. 2014, "Las muertes en la Amazonia delatan la dimensión de la tala ilegal en Perú", El País Internacional, 15 septiembre 2014, http://internacional.elpais.com/internacional/2014/09/14/ actualidad/1410712501_025673.html. 
Feather, Conrad. 2014, Violating rights and threatening lives: The Camisea gas project and indigenous peoples in voluntary isolation, Morton-in-Marsh (Reino Unido), Forest Peoples Programme.

Federación Nativa del Río Madre de Dios y Afluentes (FENAMAD). 2014, "Aislados Mashco Piro nuevamente aparecen frente a comunidad Monte Salvado". 11 septiembre 2014, https://m.facebook.com/story.php?story fbid $=824622540904783 \&$ id $=201180499915660$

Ferguson, R. Brian y Neil Whitehead. 1992, War in the Tribal Zona, NM, School of American Research Press, Santa Fe.

Fernández Moro, Wenceslao. 1952, Cincuenta Años en la Selva Amazónica, Imprenta y Litografía Juan Bravo, Madrid.

Finer, Matt et al. 2008, "Oil and Gas Projects in the Western Amazon: Threats to Wilderness, Biodiversity and Indigenous Peoples", PLoS One, http://dx.plos.org/10.1371/journal.pone.0002932.

Finer, M., y S. Novoa. 2016. "Mineros ilegales cambian el curso del Rio Malinowski (limite Reserva Nacional Tambopata)", MAAP 33, http://maaproject.org/2016/desvio-malinowski/.

2015, "Patrones y Drivers de la Deforestación en la Amazonía Peruana," MAAP Síntesis \#1, http://maaproject. org/2015/09/maap-sintesis $1 /$.

Finer, M., S. Novoa y T. Olexy. 2016a, “Gobierno Enfrenta Invasión de la Minera Aurífera en La Reserva Nacional Tambopata”, MAAP 24, http://maaproject.org/2016/tambopata2/.

2016b, "Construction of a New Highway between Manu National Park and Amarakaeri Communal Reserve (Madre de Dios)", MAAP 29, http://maaproject.org/2016/manu-road/.

2016c, "Invasión de la Reserva Nacional Tambopata se Intensifica', MAAP 30, http://maaproject.org/2016/ invasion-tambopata-2/.

Flores Tello, Eldy. 2011, "Madre de Dios tiene concesiones en la mayor parte de su territorio", 16 noviembre 2011, http://elcomercio.pe/peru/lima/madre-dios-tiene-concesiones-mayor-parte-su-territorio_1-noticia-1334405.

Global Witness. 2014, "El ambiente mortal. El aumento de asesinatos de defensores ambientales y de la tierra", London, Global Witness, Ltd., disponible en: https://www.globalwitness.org/en/campaigns/environmentalactivists/perus-deadly-environment/.

Gow, Peter. 1991, Of Mixed Blood. Kinship and History in Peruvian Amazonia, Oxford, Clarendon Press.

Grillo, Ioan. 2016, Gangster Warlords. Drug Dollars, Killing Fields, and the New Politics of Latin America, Bloomsbury Press, Nueva York.

Hill, David. 2015, "Leaked map reveals Big Gas eyeing most biodiverse place on earth", The Guardian, 24 octubre 2015, http://www.theguardian.com/environment/andes-to-the-amazon/2015/oct/24/leaked-mapreveals-big-gas-eyeing-most-biodiverse-place-on-earth.

2013, "Fears Peru's Gas Expansion will Generate Conflict in UNESCO World Heritage Site", Truthout, http:// www.truth-out.org/news/item/16129-fears-perus-gas-expansion-will-generate-conflict-in-unesco-worldheritage-site.

2011, “\$35 billion of oil plus an 'uncontacted' tribe equals cover-up," Truthout, 31 julio 2011, http://www.truthout.org/news/item/2396:35-billion-of-oil-plus-an-uncontacted-tribe-equals-coverup.

Huertas Castillo, Beatriz. 2013, "Plundered land, conflict and extinction", en: Dany R. Mahecha y Carlos y Eduardo Franky C. (Eds.) Indigenous Peoples in Voluntary Isolation and Initial Contact, International Work Group on Indigenous Affairs (IWGIA), Copenhague, pp. 56-93, http://www.iwgia.org/iwgia_files publications_files/0617_ENGELSK-AISLADOS_opt.pdf.

2010. Despojo territorial, conflicto social y exterminio. Pueblos indigenas en situación de aislamiento, contacto esporádico y contacto inicial de la Amazonía Peruana, Informe 9, International Work Group on Indigenous Affairs (IWGIA), Copenhague.

2004, "Indigenous Peoples in Isolation. Their struggle for survival and freedom", documento 100, International Work Group on Indigenous Affairs (IWGIA), Copenhague, http://www.iwgia.org/iwgia_files_publications files/0118_IsolationDoc100FullText.pdf.

Huertas Castillo, Beatriz y Alberto García (Eds.) 2003, Los pueblos indígenas de Madre de Dios: Historia, etnografia, conyuntura, International Work Group on Indigenous Affairs (IWGIA), Documento 32, Lima http://www.iwgia.org/iwgia_files_publications_files/0336_madre_de_dios.pdf.

Hydrocarbons.technology.com. 2013, "Camisea Gas Project", http://www.hydrocarbons-technology.com/ projects/camisea/.

Karl, Terri Lynn. 1997, The Paradox of Plenty, University of California Press, Berkeley.

Lawler, Andrew. 2015a, "Mashco Piro tribe emerges from isolation in Peru", Science 349 (6249), p. 679. 
Lawler, Andrew. 2015b, "Making Contact. Some of the last isolated tribes are emerging from Peru's rainforests", Science.com, http://www.sciencemag.org/news/2015/06/feature-deep-peru-s-rainforests-isolated-people-emerge.

Luna Amancio, Nelly. 2015, "Operación Amazonas: la historia del mayor decomiso de madera de origen ilegal", Ojo Público, 3 diciembre 2015, http://ojo-publico.com/132/operacion-amazonas-la-mayor-incautacion-demadera-de-origen-ilegal.

Macqueen, Angus. 2016, "Why are the lost tribes now emerging from the Amazon?", The Guardian, 20 febrero 2016, http://www.theguardian.com/world/2016/feb/21/amazon-lost-tribes-emerge-rainforestbrazil-peru.

Marris, Emma. 2016, “This Park in Peru is Nature 'in its Full Glory' - With Hunters”, National Geographic en línea, http://www.nationalgeographic.com/magazine/2016/06/manu-peru-biodiversity-national-parks.

Martins, Alexandra. 2014, "Como operan las mafias del multimillonario negocio de la madera ilegal en Perú," British Broadcasting Company, 22 septiembre 2014, Londres, http://www.bbc.com/mundo/ noticias/2014/09/140916_peru_tala_ilegal_am.

Ministerio de Cultura, Dirección General de los Derechos de Pueblos Indígenas (2013), "Resolución Viceministerial Nro. 005-2013-VMI-MC," http://www.cultura.gob.pe/sites/default/files/noticia/tablaarchivos/rvnro0092013-vmi-mc.pdf.

Moore, Thomas. 2003, "La etnografía tradicional Arakmbut y la minera aurífera”, en: Huertas Castillo y García (Eds.) 2003, Los pueblos indigenas de Madre de Dios: Historia, etnografia, conyuntura, International Work Group on Indigenous Affairs (IWGIA), Lima, pp. 58-90, http://www.iwgia.org/iwgia_files_publications_ files/0336_madre_de_dios.pdf.

Neuman, William y Andrea Zárate. 2013, "Corruption in Peru Aids Cutting of Rain Forest", 18 octubre 2013, New York Times, http://www.nytimes.com/2013/10/19/world/americas/corruption-in-peru-aids-cutting-ofrain-forest.html.

Organización Internacional del Trabajo (1989), "Convenio sobre pueblos indígenas y tribales, 1989 (núm. 169)”, http://www.ilo.org/dyn/normlex/es/f?p=NORMLEXPUB:12100:0::NO::P12100_INSTRUMENT_ ID:312314.

Organización de las Naciones Unidas. 2007. "Declaración de las Naciones Unidas sobre los derechos de los pueblos indígenas", http://www.un.org/es/events/indigenousday/pdf/indigenousdeclaration_faqs.pdf.

Pereira Gonçalves, Marilyne et al. 2012, Justice for Forests. Improving Criminal Justice Efforts to Combat Illegal Logging, Banco Mundial, Washington.

Pozzebon, Stefano. 2015, "China has crossed a major investment threshold that is going to change the entire world", Financial Times, http://business.financialpost.com/business-insider/china-has-crossed-a-majorinvestment-threshold-that-is-going-to-change-the-entire-world.

Presidente Constitucional de la República. 2008, "Aprueban Reglamento de Participación Ciudadana para la realización de Actividades de Hidrocarburos. DECRETO SUPREMO No 012-2008-EM", http://gasnatural. osinerg.gob.pe/contenidos/uploads/GFGN/DS012-2008-EM.pdf.

Pringle, Heather. 2014, "Uncontacted tribe in Brazil emerges from isolation," Science 345 (6193), p. 125, 11 July 2014.

Reider, Susan y Robert Wasserstrom. 2013, "Undermining democratic capacity: myth-making and oil development in Amazonian Ecuador", Ethics in Science and Environmental Politics 13 (1), pp. 39-47, http://www.int-res. com/abstracts/esep/v13/n1/p39-47/.

Ríos Villacorta, Alberto. 2014, "Perúpetro y la ilusión de las cifras", http://www.esan.edu.pe/conexion/ actualidad/2014/01/01/perupetro-ilusion-cifras/.

Salisbury, D. S. y C. Fagan. 2011, "Coca and conservation: cultivation, eradication, and trafficking in the Amazon borderlands", GeoJournal, http://upperamazon.org/wp-content/uploads/2011/08/ SalisburyFagan2011CocaConservation.pdf?6bd5c0.

Sanderson, Henry y Michael Forsythe. 2012, China's Superbank: Debt, Oil and Influence, Wiley, Nueva York.

Santos Granero, Fernando y Frederica Barclay. 1998, Selva central: history, economy, and land use in Peruvian Amazonia, Smithsonian Institution Press, Washington.

Shelton, Dinah. 2013, "Introduction”, en: Dany R. Mahecha y Eduardo Franky C. (Eds.) Indigenous Peoples in Voluntary Isolation and Initial Contact, International Working Group on Indigenous Affairs (IWGIA), Copenhague, pp. 8-12, http://www.iwgia.org/iwgia_files_publications_files/0617_ENGELSK-AISLADOS_ opt.pdf.

Soares de Oliveira, Ricardo. 2007, Oil and Politics in the Gulf of Guinea, Columbia University Press, Nueva York. 
Survival International. 2010, "Un niño resulta herido en un ataque de indígenas aislados", 29 octubre 2010, http:// www.survival.es/noticias/6641.

2007a, “Oil chief: Do uncontacted tribes really exist?" 22 abril 2007, http://www.survivalinternational.org/ news/2371.

2007b. "President Garcia questions existence of 'unconnected' tribes", 5 noviembre 2007, http://www. survivalinternational.org/news/2575.

s/f, "Background Briefing. Shell oil \& gas company", http://www.survivalinternational.org//about/shell

The Oil and Gas Year (TOGY). 2015, "10 years of Camisea: The natural gas revolution in Peru", 24 de febrero 2015, http://www.theoilandgasyear.com/articles/10-years-of-camisea-the-natural-gas-revolution-in-peru/.

United National Human Rights Council. 2011, Office of the High Commissioner, "Promotion and protection of all human rights, civil, political, economic, social and cultural rights, including the right to development. Report of the Special Representative of the Secretary- General on the issue of human rights and transnational corporations and other business enterprises, John Ruggie. Guiding Principles on Business and Human Rights: Implementing the United Nations 'Protect, Respect and Remedy' Framework”, http://business-humanrights. $\mathrm{org} / \mathrm{sites} /$ default/files/media/documents/ruggie/ruggie-guiding-principles-21-mar-2011.pdf

2008, Office of the High Commissioner, "Promotion and protection of all human rights, civil, political, economic, social and cultural rights, including the right to development. Protect, Respect and Remedy: a Framework for Business and Human Rights. Report of the Special Representative of the Secretary-General on the issue of human rights and transnational corporations and other business enterprises, John Ruggie", http://www. reports-and-materials.org/sites/default/files/reports-and-materials/Ruggie-report-7-Apr-2008.pdf.

Urteaga Crovetto, Patricia. 2003, "La minería y los pueblos indígenas de Madre de Dios", en: Huertas Castillo y García (Eds.) Los pueblos indígenas de Madre de Dios: Historia, etnografia, conyuntura, International Work Group on Indigenous Affairs (IWGIA), Lima, pp. 315-339, http://www.iwgia.org/iwgia_files_publications_ files/0336_madre_de_dios.pdf.

Wallace, Scott. 2015. "Some Isolated Tribes in the Amazon Are Initiating Contact", National Geographic, 13 agosto 2015, http://news.nationalgeographic.com/2015/08/150813-uncontacted-amazon-tribes-peru-brazil/.

2014, "Quadruple Homicide in Peruvian Amazon Puts Criminal Logging in Spotlight", 12 septiembre 2014, National Geographic, http://news.nationalgeographic.com/news/2014/09/140911-peru-amazon-illegallogging-chota-alto-tamaya/.

Watts, Jonathan. 2014. "Fears drug traffickers may be driving isolated tribe from Amazon home", The Guardian, 15 July 2014, http://www.theguardian.com/world/2014/jul/15/drug-traffickers-driving-isolated-jaminawatribe-amazon-home.

Wasserstrom, Robert. 2016, "Waorani Warfare on the Ecuadorian Frontier, 1885-2013, Journal of Latin American and Caribbean Anthropology (por publicarse).

2015, "China undermines democracy in Africa", The Free Press, Rockport, Maine, 12 noviembre 2015, p. 4.

Wasserstrom, Robert y Douglas Southgate. 2013, "Deforestation, Agrarian Reform and Oil Development in Ecuador, 1964-1994," Natural Resources 4, pp. 31-44, http://www.scirp.org/journal/nr.

Zárate, Andrea y William Neuman. 2014, "Peru Investigates the Killing of an Environmental Advocate", 10 septiembre 2014, New York Times, http://www.nytimes.com/2014/09/11/world/americas/peru-investigatesthe-killing-of-an-environmental-advocate.html.

Zarzar, Alonso. 2000, Tras la huellas de un antiguo presente, Defensoría del Pueblo, Lima. 